Diagnostic Accuracy Study

\title{
An Evaluation of the Diagnostic Accuracy of Liquid Chromatography-Tandem Mass Spectrometry Versus Immunoassay Drug Testing in Pain Patients
}

Amadeo Pesce, PhD, Murray Rosenthal, DO, Robert West, MS, Cameron West, PhD, Bridgit Crews, PhD, Charles Mikel, PhD, Perla Almazan, CLS, MT, and Sergey Latyshev, MS

From: Millennium Laboratories Research Institute, San Diego,

CA.

Address correspondence: Cameron West, PhD

Millennium Laboratories 16981 Via Tazon

San Diego CA 92127 E-mail:

cwest@becausepainmatters.

com

Disclaimer: There was no external funding in the preparation of this manuscript.

Conflict of interest: None.

Manuscript received: $10 / 23 / 2009$

Accepted for publication: $12 / 31 / 2009$

Free full manuscript: www.painphysicianjournal.com
Background: Immunoassay screening is used by pain physicians to determine compliance with controlled substances. Because clinical use of pain medications is different from illicit drug use, there is a need to evaluate the level of diagnostic accuracy of this procedure for the pain patient.

Objective: To compare the results of automated screening by immunoassay with analysis by Liquid Chromatography-Tandem Mass Spectrometry (LC-MS/MS) in identifying pain patients using illicit drugs and pain patients excreting low concentrations of their prescribed medications.

Study Design: A diagnostic accuracy study.

Methods: Urine samples from 4,200 pain patients were tested by immunoassay and LCMS/MS for the following drugs and metabolites: Amphetamine, Methamphetamine, Alphahydroxyalprazolam, Lorazepam, Nordiazepam, Oxazepam, Temazepam, Cannabinoids, Cocaine, Methadone, Methadone Metabolite, Codeine, Hydrocodone, Hydromorphone, Morphine, Propoxyphene, and Norpropoxyphene.

Results: In a number of patients negative immunoassay findings were superseded by positive results on analysis by Mass Spectrometry. These were termed false negative results. The greatest failures were for the benzodiazepines (28\%) and for cocaine (50\%).

Limitations: The study was limited by the lack of complete demographics for the cohort and because only one immunoassay diagnostic product was used. It was also limited because not all drugs react the same in the immunoassay.

Conclusions: We show that in general, immunoassay screening results are accurate, although as shown in this study there are many false negative observations. The use of LC-MS/MS technology significantly decreases the number of false negative results.

Key words: Drug abuse, illicit drug use, opioids, illicit drugs, benzodiazepines, false negatives, liquid chromatography-mass spectrometry, immunoassay, adherence monitoring

Pain Physician 2010; 13:273-281
W ith the increasing therapeutic use of opioids there is also increasing abuse by patients being prescribed these drugs for pain management (1-14). Non-medical use of other psychotherapeutic and illicit drugs is also prevalent in this population $(4,5)$. One way to monitor opioid adherence and identify abuse is urine drug testing $(6-8,15)$.

Physicians treating pain patients with prescription drugs that are controlled substances order drug 
screens in part to ensure compliance and determine illicit drug use $(7,8,15-19)$.

Forensic drug testing focuses on illicit drug use, the determination of which is based on positive results of urine drug tests. Negative results are considered an indication of no illicit drug use (20). The drug testing utilized by pain physicians concerns both positive and negative urine drug screening observations. In the case of prescription medications, positive results indicate compliance and negative results indicate the potential for drug diversion $(15,21)$. The sensitivity of any immunoassay is defined as the concentration of the index drug which yields a positive response. The specificity of any immunoassay is defined as the concentration of any compound which also gives a positive response (10). For example, immunoassay tests for morphine also react with hydromorphone and hydrocodone. For many commercial assays the reaction with hydromorphone and hydrocodone occurs at higher concentrations than with that of morphine. In this instance, even though the assay is specific for an opiate for all of these reactions, the sensitivity is poorer for hydromorphone and hydrocodone. Similar sensitivity and specificity considerations extend to immunoassay measurement of the various benzodiazepine drugs and their metabolites (22).

It is well known that a number of commercial assays yield false negative results $(7,8,22,23)$. There have been several studies determining the accuracy of specific drug immunoassays (24-27).

Historically, mass spectrometry procedures have been expensive to perform because they required sample extraction and derivatization of the test drug. The advent of rapid dilution and injection technology specific to Liquid Chromatography-Tandem Mass Spectrometry (LC-MS/MS) has reduced the cost of this procedure, making it possible to perform urine drug screening and confirmation rapidly and at a reasonable cost. The increased sensitivity and selectivity of this technology has created standards that reflect lower drug cutoffs and a broader, more accurate identification of illicit drug content in the urine drug test (UDT).

Fraser and Zamecivik (28) and Hattab et al (29) have shown that cutoffs below the standard Substance Abuse and Mental Health Services Administration (SAMHSA) concentrations used as cutoffs utilized by laboratories testing for illicit drugs show higher incidences of illicit drug use in their respective study test populations. Mikel et al (30) conducted a study using lower cutoffs with LC-MS/MS instruments that showed higher incidences of licit and illicit drug use among pain patient populations. However, that study was limited because it did not do a direct comparison of actual immunoassay observations versus those done by LC-MS/ MS. It remains to be seen how accurately immunoassays detect licit and illicit drug use (15).

The rationale for this study was to better define the accuracy of immunoassays compared with the gold standard LC-MS/MS analyses on the same specimens. The purpose of the study was to compare the accuracy of the detection of opioids, benzodiazepines, and propoxyphene as well as the illicit drugs marijuana, cocaine, and methamphetamine using an automated immunoassay versus detection by LC-MS/MS.

\section{Methods}

\section{Participants}

The cohort was comprised of 4,200 patients treated with opioids for chronic pain. All patients were tested as part of the usual practices of the treating physicians and no exclusion criteria were used in the selection of these patients. This research was approved by the Aspire IRB, 9320 Fuerte Dr. Suite 105, La Mesa, CA, 91941. As this study was prospective in nature, treatment of patients was not affected. Participant recruitment was the result of pain physicians deciding to test their patients for either routine drug screens or for reasons of suspicion according to the American Society of Interventional Pain Physicians (ASIPP) guidelines (7).

\section{Test Methods}

No outside funding was provided for this study. Physicians in their office practices initiated the test requests and collected the urine specimens for this study onsite. Most physicians conducted initial drug screens using point of care devices. These results were not included in the comparison presented. Rather, they were used by the physicians to select the test menu for the additional screening and confirmation testing performed at Millennium Laboratories. All data were collected at Millennium Laboratories and Millennium Laboratories Research Institute.

Sequential urine specimens sent by pain physicians were tested for the analytes requested by that physician: opioids, benzodiazepines and propoxyphene, as well as marijuana, cocaine, and methamphetamine. It should be noted that not all physicians requested the same tests. Therefore, only the results of those tests that fell into these categories were compared. Analyses were performed on each consecutive specimen by both 


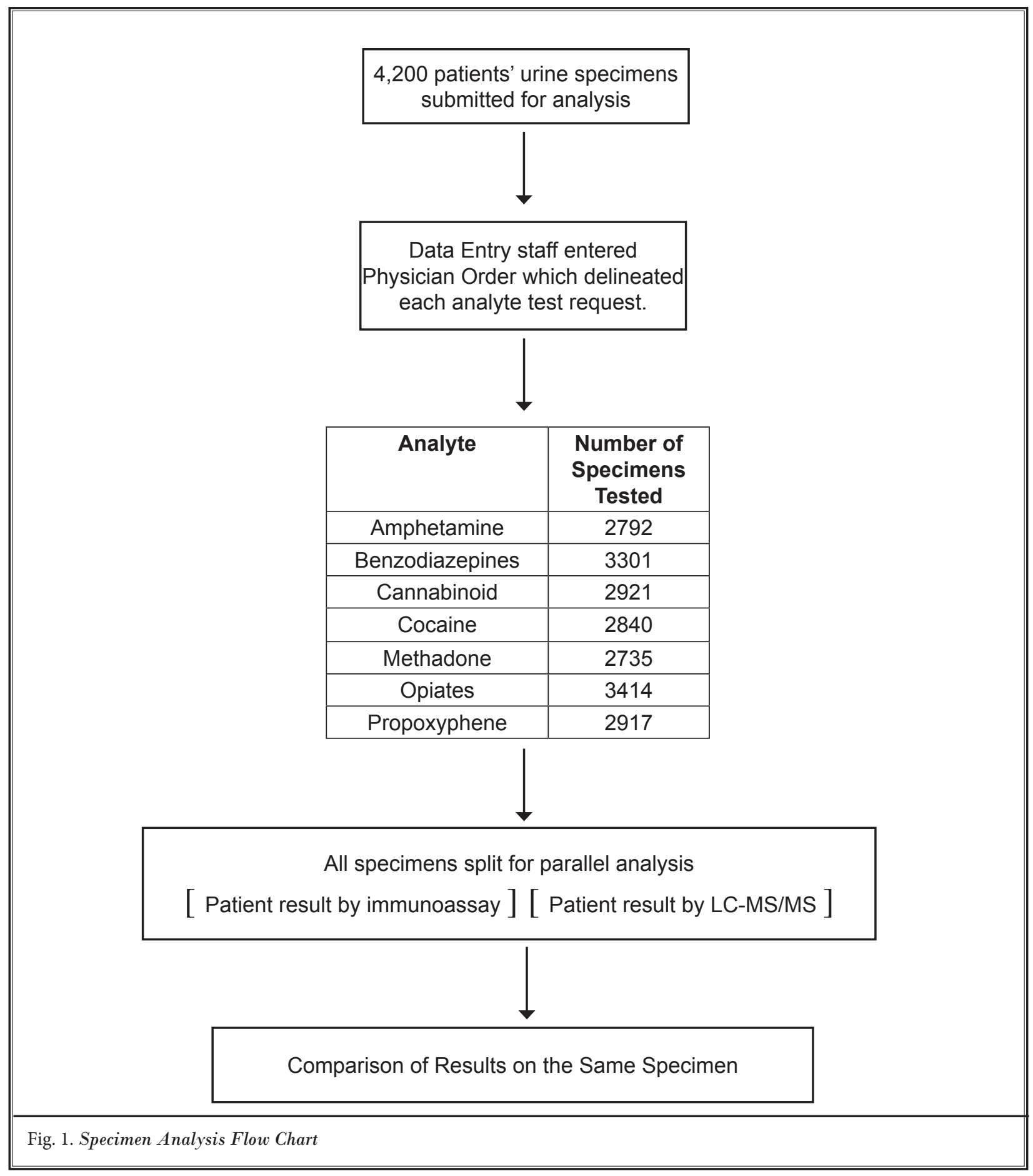

immunoassay and LC-MS/MS techniques. The tests were performed in parallel; that is, they were conducted simultaneously on split specimens (Fig. 1).

No data was collected on treatment or on severity of disease.
The immunoassay (EIA) procedures used DRI reagents from Microgenics Corporation (31). These were: DRI Amphetamine Assay, DRI Benzodiazepine Assay, DRI Cannabinoid (THC) Assay, DRI Cocaine Assay, DRI Methadone Assay, DRI Opiate 300 Assay, and DRI Pro- 
poxyphene Assay. (Microgenics Corp. 46360 Fremont Blvd., Fremont, CA 94538 USA.) They were used in the Olympus model 640 analyzer (Olympus America Inc., 3500 Corporate Parkway, P.O. Box 610 Center Valley, PA 18034-0610, USA) according to the manufacturer's (Microgenics) directions. The amphetamine, cannabinoid, cocaine, methadone, and propoxyphene assays were set up as single point calibrators. The benzodiazepine and opiate assays were set up as semi-quantitative assays. Table 1 lists the cutoff values for the Microgenics assays. Values above the calibration cutoff were defined as positive. Those below were defined as negative. Because the EIA cutoffs vary by drug, these are listed as a separate column in Table 1. The immunoassay reproducibility was $\pm 25 \%$ of the cutoff for amphetamine, marijuana, opioids, cocaine which are set by SAMHSA (32). The benzodiazepine and propoxyphene assays were the same.

The reference standard was detection and quantitation by LC-MS/MS. The LC-MS/MS procedures were performed on Agilent 6410 instruments (Agilent Corporation, 5301 Stevens Creek Blvd, Santa Clara, CA
95051,USA). The method was that described by Yang (33). Each analyte was specifically identified by the mass ion of the parent drug, 2 qualifier ions, and the ratio of the 2 qualifier ions to each other. The ratio of the parent mass ion to its deuterated internal standard was used for quantitation. The LC-MS/MS cutoffs are the lower limit of quantitation of the investigators' procedure and are listed in Table 1. Values above the Lower Limit of Quantitation (LOQ) were defined as positive. Those values below were defined as negative. The accuracy of the LC-MS/MS determinations is approximately $\pm 20 \%$ of the target value as determined by proficiency testing data. The method was validated to meet the Clinical Laboratory Improvement Amendments (CLIA) standards required by the state of New York and the College of American Pathologists confirmatory proficiency testing $(34,35)$.

The units used were nanograms per milliliter (ng/ $\mathrm{mL}$ ). The cutoffs used for the immunoassay for amphetamine, marijuana, opioids, and cocaine were those set by SAMHSA (32). The benzodiazepine and propoxyphene cutoffs are those set by the immunoassay manufactur-

Table 1 Cutoff values for EIA and LC-MS/MS

\begin{tabular}{|c|c|c|c|}
\hline Compound & EIA Cutoff (ng) & LC-MS/MS Cutoff (ng) & Relative EIA Reactivity \\
\hline Amphetamine class & 1000 & & \\
\hline Amphetamine & & 100 & 1000 \\
\hline Methamphetamine & & 100 & 1000 \\
\hline Benzodiazepine class & 200 & & \\
\hline Alpha-hydroxyalprazolam & & 20 & Not listed \\
\hline Lorazepam & & 40 & 200 \\
\hline Nordiazepam & & 40 & 150 \\
\hline Oxazepam & & 40 & 200 \\
\hline Temazepam & & 50 & 25 \\
\hline Cannabinoids & 50 & 10 & - \\
\hline Cocaine & 300 & 25 & - \\
\hline Methadone & 300 & 50 & 300 \\
\hline Methadone Metab & & 50 & 10,000 \\
\hline Opiates class & 300 & & \\
\hline Codeine & & 50 & 180 \\
\hline Hydrocodone & & 50 & 1700 \\
\hline Hydromorphone & & 50 & 4000 \\
\hline Morphine & & 50 & 300 \\
\hline Propoxyphene Class & 300 & & \\
\hline Propoxyphene & & 100 & 300 \\
\hline Norpropoxyphene & & 100 & 500 \\
\hline
\end{tabular}


er (31). The LC-MS/MS cutoffs were set by Millennium Laboratories (30). These cutoffs represent the LOQ. All of the test results were reviewed by clinical laboratory scientists certified by the State of California.

The readers of the index immunoassay tests were separated from the readers of the reference standard (LC-MS/MS) and were thus blind (masked) to the results of the other test. The only clinical information available to either set of readers was that the urines were from pain patients.

A simple tabulation was done: the number of patients undergoing the test, the number positive by immunoassay, and the number positive by LC-MS/MS. The number positive by LC-MS/MS was used to calculate the total positive number for that test. The number positive by immunoassay was defined as those positive for that test. False negatives were the difference between the 2 observations.

\section{Statistical Methods}

Data was retrieved from Millennium's laboratory information management system (LIMS) using an algorithm that recorded any LC-MS/MS results from the test panel as positive and matched those findings with the recorded EIA result. The total of all positive confirmed EIA results was generated in another report and combined with the false negative data to form Table 2 .

As LC-MS/MS was the gold standard for this study, there were no false positives on this instrumentation. False positives for immunoassay were determined by the LC-MS/MS procedure and were deleted from the calculation. For example, the opiate immunoassay gave false positive results when high concentrations of oxycodone or oxymorphone were present. These were not included in the number of LC-MS/MS positives in Table 2. The same procedure was applied to all the other analytes.

\section{Results}

This study was conducted between 10/16/08 and $11 / 04 / 08$. The distribution of the reference test results are presented in Table 2. As the tests were performed on split samples, there were no missing results. No adverse events were observed from performing either test. The $99 \%$ confidence intervals for the immunoassays were $\pm 25 \%$ of the cutoff. Test reproducibility at greater than $25 \%$ above or below the immunoassay cutoffs were greater than $99 \%$. The $95 \%$ confidence limits for the LCMSMS cutoffs were $\pm 30 \%$. There were no indeterminate results.

The total tests for each separate analyte represented $100 \%$ for that sample. ElA positive was defined as a positive test result by immunoassay. An LC-MS/MS positive result was defined as that value above the cutoff standard defined in Table 1. The difference between the positive findings for any given analyte in the immunoassy and the LC-MS/MS were defined as a False Negative. The Positive Rate was defined as the percentage of tested samples found positive by LC-MS/MS. The Percent False Negative was derived by dividing the number of LC-MS/MS positive results by the false negative number for that drug times $100 \%$.

The difference between the immunoassay and LCMS/MS cutoffs as listed in Table 1 accounted for all of the false negative results for the amphetamine, methamphetamine, cannabinoid, cocaine, and methadone analytes. The immunoassays for the benzodiazepines and the opioids were designed to be positive or negative for the entire class of drugs. However, each drug or metabolite can react differently. These are listed in Table 1 as the relative reactivity for each benzodiazepine metabolite. For some of them, more than one metabolite was present; for others the nominal EIA reactivity is for the un-conjugated form of the drug, not

Table 2. Positive and false negative test rates for drugs in urines of pain patients

\begin{tabular}{||l|c|c|c|c|c|c||}
\hline \multicolumn{1}{|c|}{ Drug } & $\begin{array}{c}\text { Total } \\
\text { Tested }\end{array}$ & EIA Positive & $\begin{array}{c}\text { LC-MS/MS } \\
\text { Positive }\end{array}$ & $\begin{array}{c}\text { Difference } \\
\text { (False Negative) }\end{array}$ & $\begin{array}{c}\text { Positive } \\
\text { Rate (MS) }\end{array}$ & $\begin{array}{c}\text { Percent False } \\
\text { Negative }\end{array}$ \\
\hline Amphetamine & 2792 & 137 & 151 & 14 & $5.4 \%$ & $9.3 \%$ \\
\hline Benzodiazepines & 3301 & 998 & 1278 & 280 & $38.7 \%$ & $22 \%$ \\
\hline Cannabinoid & 2921 & 370 & 414 & 44 & $14.1 \%$ & $10.6 \%$ \\
\hline Cocaine & 2840 & 64 & 128 & 64 & $4.5 \%$ & $50 \%$ \\
\hline Methadone & 2735 & 258 & 275 & 17 & $10 \%$ & $6.1 \%$ \\
\hline Opiates & 3414 & 2191 & 2233 & 42 & $65 \%$ & $1.9 \%$ \\
\hline Propoxyphene & 2917 & 204 & 264 & 62 & $9.1 \%$ & $23.4 \%$ \\
\hline
\end{tabular}


the excreted form. Since it was not possible to compare results for benzodiazepines and hydrocodone and hydromorphone at the nominal cutoff, this is a limitation of the study. However, in virtually all of the false negative cases of the benzodiazepines and opioids, the observed mass spectrometry values exceeded the nominal cutoff. That is, the false negative observation was due to the poor cross-reactivity of the immunoassay and not to the cutoffs.

The 14 false negative EIA amphetamine observations were due to the differences in cutoffs between immunoassay and LC-MS/MS. The 44 false negatives for cannabinoids, 64 for cocaine, and 17 for methadone were due to the same factor.

Virtually all of the 42 false negative opiate results were in urines containing hydromorphone and hydrocodone. This is consistent with the poor cross reactivity of these compounds compared to morphine in the EIA (31). The 62 false negative results for propoxyphene were almost all due to the presence of norpropoxyphene without detectable propoxyphene. According to the nominal cross reactivity listed in the package insert, at least $40 \%$ of these should have been EIA positive (31). The 280 false negative values for benzodiazepines were almost all due to the presence of lorazepam. The investigators observed LC-MS/MS lorazepam values that in many cases far exceeded the nominal EIA cutoff listed in the package insert (31). The large number of benzodiazepine false negative values was due to poor cross reactivity with the excreted metabolites and not to the difference between cutoffs for the EIA and LCMS/MS procedures.

\section{Discussion}

The results of this study show that although immunoassays do provide correct results in most cases, they are not always able to detect either illicit drugs or the excretion products of drugs used in the treatment of pain patients. For example, in this study assays for amphetamines, cannabinoids, cocaine metabolite, and methadone worked well at the nominal cutoffs. However, a significant number of cases were observed where the quantities of these substances were between the nominal EIA cutoff and the LOQ of the LCMS/MS. Additionally, we observed the poor reactivity of hydromorphone and hydrocodone by immunoassay which indicated that the nominal opiate cutoff of 300 ng for EIA was misleading, albeit this nominal cutoff is correct for morphine and codeine. Although false negatives for the opioids occurred in only $2 \%$ of the tests, this represents an important failure of EIA testing, since the repercussions for a patient who is discharged from a practice can be life-changing. All of this is in line with Trescot et al (7) who succinctly stated that, "Since false negatives and false positives are possible, when questions arise, prior to taking any actions a confirmatory test or no threshold test must be performed in the laboratory."

One limitation of this study is the lack of complete demographic information on the cohort. However, Manchikanti et al (1) have outlined the usual description of this patient population. The study is also limited because only one manufacturer's set of products was used. However, in White and Black's book, Pain Management Testing Reference, they list all the package inserts immunoassay variances for opioids and benzodiazepines (22). These listed antibody cross-reactivities are similar to those listed in the Microgenics assays package inserts. Therefore, we believe the results reported here are applicable to other instruments and drug testing reagents.

This study revealed a large false negative finding for propoxyphene. Analysis by LC-MS/MS revealed $23.4 \%$ more patients taking propoxyphene than were revealed when tested by EIA. This large variation appeared to be due to the poor reactivity of the norpropoxyphene with this immunoassay. Equally disconcerting was the investigators' finding of a $22 \%$ false negative rate for the benzodiazepine assay. Almost all could be ascribed to the poor cross reactivity with lorazepam.

It is well known that there is a large variance in the immuno-reactivity with different benzodiazepines $(25,26)$. As described in one of the package inserts, the cross reactivity of the benzodiazepine glucuronides is less than that of the parent drug. Specifically, for CEDIA assays, the lorazepam glucuronide reacts $4 \%$ as well as the parent drug. The poor cross reactivity of lorazepam has previously been described (25). As observed here, false negative results by immunoassay for benzodiazepines can be expected. It is surprising that the manufacturer of the benzodiazepine immunoassay has not corrected this deficiency.

The investigators' patient population had an illicit drug observation for cocaine of about $2.25 \%$ by the immunoassay screening method and $4.5 \%$ by the LCMS/MS method. Cone et al (36) observed an incidence of cocaine of $3.2 \%$ in their pain population. It appears then that the populations are roughly equivalent. Therefore, the investigators argue that the incidence of cocaine abuse may have been much higher in Cone's 
population but went undetected because of their use of a higher screening cutoff in that study. If the new National Library Certification Program guidelines were used, a higher incidences would certainly have been observed (37).

Even though the effectiveness of opioids in the treatment of chronic non-cancer pain has limited evidence, these medications are widely prescribed (38). Because of the possibility of abuse and diversion, urine drug screening is used as a physician tool to monitor these patients. The presence or absence of substances in their urine guides them in the patient's treatment $(7,8)$. Negative results on a urine drug screen imply non-compliance with a physician's prescribed medications, often causing the physician to conclude that the patient is diverting drugs (39). This may not always be a correct conclusion though. For example, a patient may have been taking the prescribed drug and the excreted urine concentrations were below the cutoff value used for the test. This would result in a false negative. Another problem could occur when a patient taking illicit drugs excretes at test time below the cutoff level $(7,8)$. Whether this is due to the patient having taken only a small amount of the drug prior to the test, having last used it several days prior to the test, or metabolizing in an unusual way, the knowledge that the patient is taking illicits still eludes the doctor.

Misinterpretation of lab results by the physician can also be problematic if the doctor lacks sufficient understanding of the pharmacology of opioids $(7,8,15,40$ 42). For example, if the physician doesn't know that hydormorphone is the excreted metabolite of morphine - and this study showed that hydromorphone reacts poorly with the immunoassay antibody - then he or she might believe that the drug test result of negative for opioids means that the patient is not taking their morphine when in fact they are.

Many physicians may not be aware of the variance in sensitivity of assays used by laboratories to analyze urine specimens for the presence of prescription medications. They may assume that a negative result indicates non-compliance. Again, the potential consequence to the patient is grave. This raises the question: Is it the responsibility of the laboratory to carefully instruct the physician as to the limit of their testing? This study leads these investigators to believe that it is. The National Academy of Clinical Biochemistry suggests that when immunoassays are used, "the laboratory should list the major cross-reacting substances for each drug class when a positive result is reported. It may also be appropriate to indicate in the final report (e.g. "notes" section) that a negative urine drug result does not indicate absence of all drugs of abuse" (43).

SAMHSA sets the guidelines for detecting illegal drug use in Department of Transportation workers and these have been adopted for workplace testing (37). These cutoffs are useful for emergency unit testing of potentially overdosed patients. In these cases, the urine concentrations of drugs are very high and overcome the limitations of sensitivity. These same guidelines have been applied to the detection of drugs in the pain patient population $(7,36)$. Leading pain physicians recognize that this is not appropriate: "Clinicians should remember that the cutoff concentrations used for drugs in federally regulated testing, particularly opioids, are too high to be of value in clinical practice" (7).

Substance abuse is a well-known problem in the pain patient population (1-8). Bearing in mind that the present investigators detected double the number of illicit cocaine users by LC-MS/MS using a lower cutoff compared to those observed by immunoassay using SAMHSA cutoff, this prompts another question: Should physicians treating pain patients go beyond the legal guidelines and demand more accurate testing using lower cutoffs such as those provided by LC-MS/MS? Again, these investigators believe so.

The implications are significant, not only for pain doctors and their patients, but for society as well. According to the National Prescription Drug Threat Assessment, diversion and supplementation (the acquisition of prescription drugs for recreational use) costs public and private insurers $\$ 72.5$ billion per year for 6.9 million non-medical prescription drug abusers $(44,45)$. This is over $\$ 10,000$ per abuser per year.

It is believed that more than $10 \%$ of pain patients abuse medications $(3,13)$. The problem for physicians is to detect the abusers among their patients. This is not easy. It has been shown that even experienced doctors are often fooled by pain patients who are abusing medications. Researchers at Cornell University found that physicians detect a bogus patient only $10 \%$ of the time, even when warned of a visit by an actor with a "pain" condition; in addition, physicians were liable to mistakenly identify real patients as actors (46). Therefore, it could be surmised that subjecting pain patients to urine drug testing at lower quantitative levels could expose some of those abusers who have heretofore gone undetected, substantially decreasing their cost to society. 
It is important to consider why there is a difference in accuracy between screening immunoassays and confirmation by LC-MS/MS. First, cutoffs for are higher for the immunoassay procedure than mass spectrometry (7). Second, the 2 techniques measure different compounds. Immunoassay procedures measure the glucuronide (metabolized) form of the drug, whereas confirmation methods measure the drug itself. Thus, it is to be expected that there are differences in accuracy between immunoassay and mass spectrometry procedures.

\section{LIMITATIONS}

The study was limited by the lack of complete demographics for the cohort and because only one immunoassay diagnostic product was used. It was also limited because not all drugs react the same in the immunoassay.

\section{Conclusions}

Many clinical and forensic evaluations of excreted drugs are dependant on initial screening results. We show that in general, immunoassay screening results are accurate, although as shown in this study, there are many false negative observations. The use of LC-MS/MS technology significantly decreases the number of false negative results. This finding reinforces the importance to both physician and patient of accurate urine drug testing. This study shows that testing by LC-MS/MS should be considered the standard of choice for analysis of drugs in urine.

\section{Acknowledgments}

We would like to thank the editorial board of Pain Physician for review and criticism in improving the manuscript.

\section{References}

1. Manchikanti L, Singh A. Therapeutic opioids: A ten-year perspective on the complexities and complications of the escalating use, abuse, and nonmedical use of opioids. Pain Physician 2008; 11: S63-88.

2. Manchikanti L, Damron KS, McManus C, Barnhill R. Patterns of illicit drug use and opioid abuse in patients with chronic pain at initial evaluation: A prospective observational study. Pain Physician 2004; 7:431-437.

3. Kuehn B. Efforts aim to curb opioid deaths, injuries. JAMA 2009; 301:12131215.

4. Manchikanti L, Cash KA, Damron KS, Manchukonda R, Pampati V, McManus CD. Controlled substance abuse and illicit drug use in chronic pain patients: an evaluation of multiple variables. Pain Physician 2006; 9:215-226.

5. Manchikanti L, Manchukonda R, Pampati V, Damron KS Evaluation of abuse of prescription and illicit drugs in chronic pain patients receiving short acting (hydrocodone) or long acting (methadone) opioids. Pain Physician 2005; 8:257-261.

6. Manchikanti L., Manchukonda R., Pampati V., Damron KS, Brandon DE, Cash KA, McManus CD. Does random urine drug testing reduce illicit drug use in chronic pain patients receiving opioids? Pain Physician 2006; 9:123-129.
7. Trescot AM, Helm S, Hansen H, Benyamin, R, Glaser S, Adlaka R, Patel S, Manchikanti L. Opioids in the management of chronic non-cancer pain: An update of American Society of the Interventional Pain Physicians' (ASIPP) Guidelines. Pain Physician 2008; 11:S5S62.

8. Manchikanti L, Atluri S, Trescot AM, Giordano J. Monitoring opioid adherence in chronic pain patients: Tools, techniques, and utility. Pain Physician 2008; 11:S155-S180.

9. Smith HS, Deer TR, Staats PS, Singh V, Sehgal N, Cordner H. Intrathecal drug delivery. Pain Physician 2008; 11:S89S104.

10. Benyamin R, Trescot AM, Datta S, Buenaventura RM, Adlaka R, Sehgal N, Glaser SE, Vallejo R. Opioid complications and side effects. Pain Physician 2008; 11:S105-S120.

11. Silverman S. Opioid induced hyperalgesia: Clinical implications for the pain practitioner. Pain Physician 2009; 2:679-684.

12. Manchikanti KN, Manchikanti L, Damron KS, Pampati V, Fellows B. Increasing deaths from opioid analgesics in the United States: An evaluation in an interventional pain management practice. $J$ Opioid Manage 2008; 4:271-283.

13. Hall AJ, Logan JE, Toblin RL, Kaplan JA, Kraner JC, Bixler D, Crosby AE, Paulozzi
LJ. Patterns of abuse among unintentional pharmaceutical overdose fatalities. JAMA 2008; 300:2613-2620.

14. McLellan AT, Turner B. Prescription opioids, overdose deaths, and physician responsibility. JAMA 2008; 300:26132620.

15. Manchikanti L, Malla Y, Wargo BW, Cash KA, Pampati V, Damron KS, McManus $\mathrm{CD}$, Brandon DE. Protocol for accuracy of point of care (POC) or in-office urine drug testing (immunoassay) in chronic pain patients: A prospective analysis of immunoassay and liquid chromatography tandem mass spectometry (LC/MS/ MS). Pain Physician 2010; 13:E1-E22.

16. Gilbert JW, Wheeler GR, Mick GE, Storey $B B$, Herder SL, Richardson GB, Watts E, Gyarteng-Dakwa K, Marino BS, Kenney $C M$, Siddiqi M, Broughton PG. Urine drug testing in the treatment of chronic noncancer pain in a Kentucky private neuroscience practice: The potential effect of Medicare benefit changes in Kentucky. Pain Physician 2010; 13:187-194.

17. Gilbert JW, Wheeler GR, Mick GE, Storey BB, Herder SL, Richardson GB, Watts E, Gyarteng-Dakwa K, Marino BS, Kenney CM, Siddiqi M, Broughton PG. Importance of urine drug testing in the treatment of chronic noncancer pain: Implications of recent Medicare policy changes in Kentucky. Pain Physician 2010; 13:167-186. 
18. Pesce A, West C, Rosenthal M, West R, Crews B, Mikel C, Almazan P, Latzshev S. Marijuana correlates with use of other illicit drugs in a pain patient population. Pain Physician 2010; 13:?????

19. West R, Pesce A, West C, Crews B, Mikel C, Almazan P, Rosenthal M, Latyshev S. Comparison of clonazepam compliance by measurement of urinary concentration by immunoassay and LC-MS/MS in pain management population. Pain Physician 2010; 13:71-78.

20. Department of Health and Human Services. Substance Abuse and Mental Health Services Administration. Mandatory Guidelines for Federal Workplace Drug Testing Programs: Notice. Federal Register, 2009; 73: 228.

21. Hammett-Stabler C, Pesce A, Cannon D. Urine drug screening in the medical setting. Clin Chim Acta 2002; 315:125135.

22. White, RM, Black ML. Pain Management Testing Reference. AACC Press Washington, DC, 2008; pp 204-208

23. Wingert WE. Lowering cutoffs for initial and confirmation testing for cocaine and marijuana: Large-scale study of effects on the rates of drug-positive results. Clin Chem 1997; 43:100-103.

24. Valentine J, Middleton R, Sparks C. Identification of urinary benzodiazepines and their metabolites: Comparison of automated HPLC and GC-MS after immunoassay screening of clinical specimens. Journal of Analytical Toxicology 1996; 20:416-424.

25. Meatherrall R, Fraser AD. Comparison of four immunoassays for the detection of lorazepam in urine. Ther Drug Monit 1998; 20:673-675.

26. Fraser AD Psychotropic agents: The benzodiazepines. In Shaw LM, Kwong TC, Rosano TG, Orsolak PJ, Wolf BA, \& Magnani B, Eds. The Clinical Toxicology Laboratory. Contemporary Practice of Poisoning Evaluation. AACC Press, Washington, DC, 2001.

27. Kurisaki $E$, Hayashida $M$, Nihira $M$, Ohno Y, Mashiko H, Okano T, Niwa S,
\& Hiraiwa K. Diagnostic performance of triage for benzodiazepines: Urine analysis of the dose of therapeutic cases. I Anal Toxicol 2005; 29:539-543.

28. Fraser AD, Zamecnik J. Impact of lowering the screening and confirmation cutoff values for urine drug testing based on dilution indicators. Ther Drug Monit 2003; 25:723-727.

29. Hattab EM, Goldberger BA, Johannsen LM, Kindland PW, Ticino F, Chronister CW, Bertholf RL. Modification of screening immunoassays to detect sub-threshold concentrations of cocaine, cannabinoids, and opiates in urine: Use for detecting maternal and neonatal drug exposures. Ann Clin Lab Sci 2000; 30:85 91.

30. Mikel C, Almazan P, West R, Pesce A. West C, Latyshev S, Crews B. LC-MS/ MS extends the range of drug analysis in pain patients. Ther Drug Monit 2009; 31:746-748.

31. Microgenics Corp. DRI Applications Olympus AU640. 46360 Fremont Blvd., Fremont, CA 94538 USA.

32. Federal Register /Vol. 69, No. 71 /Tuesday, April 13, 2004 Mandatory Guidelines and Proposed Revisions to Mandatory Guidelines for Federal Work place Drug Testing Programs; Notices Section 2.4 Laboratory Analysis Procedures (f) (1) confirmatory drug test.

33. Analysis of Opiates using the Agilent 6410 Triple Quadrupole LC/MS/MS Oregon Medical Laboratories Contact: Grant D. Yanan Yang Agilent Technologies, Inc. Santa Clara, CA Agilent reference QQQ OML_Opiates_20070625.

34. State of New York Dept. of Health. Analytical Toxicology Proficiency Testing Program, 2008-2009. Wadsworth Center, Governor Nelson A. Rockefeller Empire State Plaza, Albany, NY.

35. AACC/CAP. 2009 Surveys and Anatomic Pathology Education Programs In: $\mathrm{Pa}$ thologists CoA, ed: College of American Pathologists; Chicago, 2009. p.80.

36. Cone EJ, Caplan YH, Black DL, Moser F. Urine drug testing of chronic pain pa- tients: Licit and illicit drug patterns. J Anal Toxicol 2008; 32:530-543.

37. Mitchell J. NLCP Implementation of the Revised Mandatory Guidelines 2010. 2009; http://74.125.95.132/ search?q=cache:i6RiZRr5-20J: https://nac.samhsa.gov/DTAB/Presentations/Juneog/JohnMitchellPTDTAB06.02-508.ppt+nlcp+guidelines\&c $\mathrm{d}=4 \& \mathrm{hl}=\mathrm{en} \& \mathrm{ct}=\mathrm{clnk} \& \mathrm{gl}=\mathrm{us}$. Accessed $10 / 20 / 2009$.

38. Trescot AM, Glaser SE, Hansen $\mathrm{H}, \mathrm{Be}-$ nyamin R, Patel S, Manchikanti L. Effectiveness of opioids in the treatment of chronic non-cancer pain. Pain Physician 2008; 11:S181-S200.

39. Kan D. Medication Diversion Reduction 2009; www.csam-asam.org/pdf/misc/ slides_Kan_Reducing_Diversion_of Medication.pdf. Accessed 10/20/2009.

40. Trescot AM, Datta S, Lee M, Hansen H. Opioid pharmacology. Pain Physician 2008; 11:S133-S153.

41. Smith HS. Peripherally-acting opioids. Pain Physician 2008; 11:S121-S132.

42. Patel V, Manchikanti L, Singh V, Schultz DM, Hayek SM, Smith HS. Systematic review of intrathecal infusion systems for long-term management of chronic non-cancer pain. Pain Physician 2009; 12:345-360

43. Wu A, McKay C. Recommendations for the use of laboratory tests to support poisoned patients who present to the emergency department Clinical Chemistry 2003; 49:357-379.

44. National Prescription Drug Threat Assessment 2009. www.usdoj.gov/ndic/ pubs33/33775/33775p.pdf. Accessed 8/18/09.

45. Results from the 2007 National Survey on Drug Use and Health: http:// oas.samhsa.gov/nsduh/2k7nsduh/ 2k7Results.pdf. Accessed 8/18/09.

46. Jung B, Reidenberg MM. Physicians being deceived. Pain Med 2007; 8:433437. 
